\title{
Updated numerical analysis of eV Seesaw with Four Generations
}

\section{Fei-Fan Lee*†}

Department of Physics, National Taiwan University, Taipei, Taiwan 10617, R.O.C.

E-mail: f1117@phys.ntu.edu.tw

\begin{abstract}
We extend the so-called "eV seesaw" scenario, where $M_{R}$ is of $\mathrm{eV}$ order, to four lepton generations. The fourth generation gives a heavy pseudo-Dirac neutral lepton, which largely decouples from other generations and is relatively stable. The framework naturally gives 3 active and 3 sterile neutrinos. We update a previous numerical analysis of a $3+3$ study of the LSND anomaly, taking into account recent results from the MiniBooNE experiment. In particular, we study the implications for the third mixing angle, as well as $\mathrm{CP}$ violation.
\end{abstract}

The 2009 Europhysics Conference on High Energy Physics,

July 16 - 222009

Krakow, Poland

\footnotetext{
* Speaker.

${ }^{\dagger}$ We thank George Hou for collaboration. This work is supported by the National Science Council of Taiwan.
} 


\section{Introduction}

Neutrino data hint at the possibility of more than three massive, mostly active neutrinos. The LSND result of $P\left(\bar{v}_{\mu} \rightarrow \bar{v}_{e}\right)=0.264 \%$ [1] can be explained if 1 sterile neutrino with $m^{2} \sim 1 \mathrm{eV}^{2}$ exists. In 2006, Hou and Soddu extended the so-called "eV seesaw" scenario [2], where the righthanded neutrino Majorana scale $M_{R}$ is of $\mathrm{eV}$ order, to four lepton generations [3]. The fourth generation gives a heavy pseudo-Dirac neutral lepton, which largely decouples from the other generations, and is relatively stable. The framework naturally gives 3 active and 3 sterile neutrinos (3+3). In 2007, the MiniBooNE (MB) collaboration presented [4] the first results of a search for $v_{\mu} \rightarrow v_{e}$ oscillations motivated by the interpretation in terms of $\bar{v}_{\mu} \rightarrow \bar{v}_{e}$ oscillations of the $\bar{v}_{e}$ excess observed in LSND. Taking this new result into account, Maltoni and Schwetz [5] found the $3+2$ scheme can fit both LSND and MB data, and at the same time account for the excess of low energy events in MB data. Here, we update the previous numerical analysis of the $3+3$ study of the LSND anomaly, taking into account MB data. In particular, we study the implications for the third mixing angle, and $\mathrm{CP}$ violation.

\section{Updated Numerical Analysis}

In a recent work [5], Maltoni and Schwetz discuss different oscillation frameworks with one or more sterile neutrinos at $\mathrm{eV}$ scale, considering global short-baseline (SBL) data. Their predicted value of the LSND probability is $P\left(\bar{v}_{\mu} \rightarrow \bar{v}_{e}\right)=0.2637 \%$. For our updated analysis, we take this value and build the $\chi^{2}$ using the latest 3 -flavor neutrino oscillation parameters $m_{j i}^{2}$ and $\sin ^{2} \theta_{i j}$ taken from Ref. [6], and require $m_{41}^{2} \sim 1 \mathrm{eV}^{2}$, a total of 7 inputs. After minimizing the $\chi^{2}$, we obtain the values for the 12 parameters $r_{i j}, i$ and $s_{i}$ [3] of the model at the best fit point, and find

$$
\chi_{\min , 2006}^{2}-\chi_{\min , 2009}^{2}=1.37 \text {, }
$$

where $\chi_{\min , 2006}^{2}$ is the $\chi^{2}$ minimum for best fit values in 2006, and $\chi_{\min , 2009}^{2}$ is the $\chi^{2}$ minimum for best fit values we get now. We conclude that agreement between eV Seesaw with 4 generations scenario and experimental data seems to improve slightly.

Fig. 1 is contour plot of $\chi^{2}$ vs $s_{1}, s_{3}$, where $s_{i}$ are rotation parameters of charged lepton sector. As $s_{2}$ is mainly determined by $\sin ^{2} \theta_{23}$, we fix $s_{2}$ at 0.9999 . Otherwise, the remaining parameters are fixed at the best fit values. In Fig. 2 we plot the four quantities $P\left(\bar{v}_{\mu} \rightarrow \bar{v}_{e}\right), \sin ^{2} \theta_{12}, \sin ^{2} \theta_{23}$ and $\sin ^{2} \theta_{13}$ vs $s_{1}$ and $s_{3}$, for the solution on the lower right of Fig. 1 . We see that $P\left(\bar{v}_{\mu} \rightarrow \bar{v}_{e}\right)$ can reach $0.2 \%$ and $\sin ^{2} \theta_{12}$ is well within range. To push $\sin ^{2} \theta_{13}$ beyond 0.02 , however, $\sin ^{2} \theta_{23}$ seems to wander away from maximal mixing of 0.5 , and values at $\sim 0.4$ or 0.6 has to be tolerated.

Finally, we study CP violation by introducing CPV phase $\delta$ to leptonic mixing matrix and find that introducing $\delta$ leads to the relative improvement of the fit of $\chi_{\min , 2009}^{2}-\chi_{\min , \mathrm{CP}}^{2}=0.007$. Thus, the quality of the global fit does not improve much by introducing $\delta$. CPV data would be needed. The case of MB antineutrino data is under study.

\section{Conclusion}

The LSND anomaly could indicate right-handed neutrino mass at eV scale, and the $3+2$ scheme can account for all data plus MB (neutrino) and LSND. A sequential 4th generation needs 


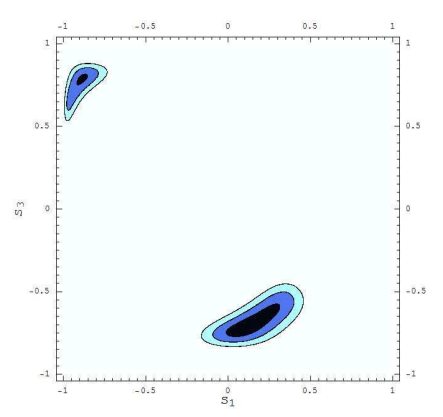

Figure 1: Contour-plot of $\chi^{2}$ vs the mixing angles $s_{1}$ and $s_{3}$, with $s_{2}=0.9999$ held fixed. The regions in different shades are only indicative, and should not be interpreted as the 1,2 and 3 regions, as the rest of the parameters are fixed at the best fit values.
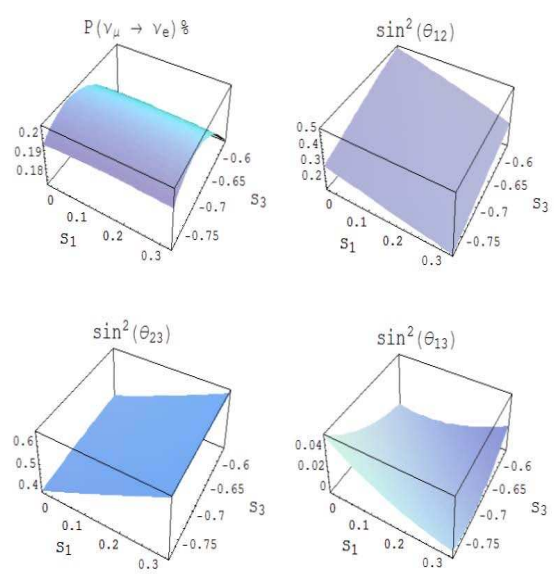

Figure 2: $P\left(\bar{v}_{\mu} \rightarrow \bar{v}_{e}\right), \sin ^{2} \theta_{12}, \sin ^{2} \theta_{23}$ and $\sin ^{2} \theta_{13}$ vs $s_{1}$ and $s_{3}$, corresponding to the lower right solution in Fig. 1.

scenarios like eV-Seesaw, and incompatible with traditional high scale Seesaw. It seems that eV Seesaw with 4 generations can accommodate LSND plus MB, indicating $\sin ^{2} \theta_{13}$ could be less than 0.02 , and the agreement with experimental data is better than before.

\section{References}

[1] LSND Collaboration, A. Aguilar et al., Phys. Rev. D 64, 112007 (2001).

[2] A. de Gouvêa, Phys. Rev. D 72, 033005 (2005).

[3] W. S. Hou and A. Soddu, Phys. Lett. B 638, 229 (2006) [arXiv:hep-ph/0512278].

[4] A. Aguilar-Arevalo et al. (MiniBooNE Collaboration), Phys. Rev. Lett. 98, 231801 (2007).

[5] M. Maltoni and T. Schwetz, Phys. Rev. D 76, 093005 (2007) [arXiv:hep-ph/0705.0107].

[6] T. Schwetz et al., New J. Phys. 10, 113011 (2008). 